\title{
A NONEMBEDDING RESULT FOR COMPLEX GRASSMANN MANIFOLDS
}

\author{
by S. G. HOGGAR
}

(Received 1st October 1969)

\section{Introduction}

A smooth map $f: M \rightarrow R^{n+k}$ of a differentiable $n$-manifold into Euclidean $(n+k)$-space is called an immersion if its Jacobian has rank $n$ at each point of $M$. If $f$ is also $1-1$, it is called an embedding.

The embedding and immersion properties of projective spaces have been widely investigated (see for example (1), (7)). We generalise by considering the complex Grassmann manifold $G_{2}\left(C^{n}\right)$ of 2-planes in $C^{n}$. This is a compact differentiable manifold of dimension $d=4 n-8$, so by the classical results of Whitney $(8,9)$

$$
G_{2}\left(C^{n}\right) \subset R^{2 d}, \quad G_{2}\left(C^{n}\right) \subseteq R^{2 d-1},
$$

where the first denotes an embedding and the second an immersion. We obtain a lower bound by proving

Main Theorem 1.1.

$$
G_{2}\left(C^{n}\right) \notin R^{d+\frac{1}{2} d}, \quad G_{2}\left(C^{n}\right) \nsubseteq R^{d+\frac{1}{2} d-1} .
$$

\section{Notation and preliminaries}

Henceforth we abbreviate $G_{2}\left(C^{n}\right)$ to $G_{n}$. Tangent and normal bundles will be denoted by $\tau$ and $v$ respectively. The canonical bundle $\gamma$ has as fibre over each point $p$ in $G_{n}$ the points of the 2-plane $p$. Taking the orthogonal complement in $C^{n}$ for each $p$ gives the complementary canonical bundle $\lambda$, with $\gamma \oplus \lambda=n$, the trivial bundle of dimension $n$.

If $\xi$ is a real $m$-plane bundle, we can tensor each fibre with $C$ over $R$ to obtain a complex $m$-plane bundle called the complexification $c(\xi)$. In the reverse direction, the decomplexification $r(\eta)$ of a complex bundle $\eta$ is realised by treating each fibre as a real space of twice the dimension. The dual of a real or complex bundle $\xi$ is written $\xi^{*}$.

We denote the total Chern class of a complex $m$-plane bundle $\eta$ by

$$
C(\eta)=1+c_{1}(\eta)+c_{2}(\eta)+\ldots+c_{m}(\eta)
$$

where the $i$ th Chern class $c_{i}(\eta)$ is an element of the cohomology of the base space of $\eta$, in dimension $2 i$.

There is the Whitney formula for two complex bundles

$$
C\left(\eta \oplus \eta^{\prime}\right)=C(\eta) . C\left(\eta^{\prime}\right)
$$


The $i$ th Pontrjagin class of a real bundle $\xi$ is defined as

$$
p_{i}(\xi)=(-1)^{i} c_{2 i}(c(\xi))
$$

We prove Theorem 1.1 by a calculation on the Chern classes of the complexified normal bundle of $G_{n}$, applied to

Proposition 2.2 (1, p. 132). Let $M$ be a differentiable m-manifold with normal bundle $v(M)$. If $p_{k}(v(M)) \neq 0$ then

$$
M \nsubseteq R^{m+2 k}, \quad M \notin R^{m+2 k-1} .
$$

For an exposition of vector bundles and Chern classes, see (3) or (5).

\section{Cohomology of $G_{n}$}

Recall that $\gamma$ is the canonical bundle over $G_{n}$, with complement $\lambda$. Write for convenience $x=-c_{1}(\gamma), y=-c_{2}(\gamma), v_{i}=c_{i}(\lambda)$. Then applying the Whitney formula (2.1) to $\gamma \oplus \lambda=n$, we have

$$
v_{0}=1, v_{1}=x, \quad v_{i}=x v_{i-1}+y v_{i-2}(2 \leqq i \leqq n) .
$$

It follows from Borel (2) that $H^{*}\left(G_{n}\right)$ is generated as a ring with 1 by $x$ and $y$, subject only to the relations $v_{n}=0=v_{n-1}$ expressed in terms of $x$ and $y$. For our computation we require an additive basis, and this is provided for by a formally identical calculation in terms of $K$-theory ((4), 4.4, 4.6) which shows:

Proposition 3.1. $H^{*}\left(G_{n}\right)$ is generated as a free abelian group by the monomials $x^{5} y^{s}$ of degree $\leqq n-2$. Monomials of degree $n-1$ are given in terms of the generators by

where

$$
v_{r} y^{n-1-r}=0(0 \leqq r \leqq n-1),
$$

$$
\begin{gathered}
v_{r}=-\Sigma\left(\begin{array}{c}
r-s \\
s
\end{array}\right) x^{r-2 s} y^{s}, \text { summing over } 0 \leqq s \leqq \frac{1}{2} r ; \\
v_{0}=1, v_{1}=x, v_{l}=x v_{i-1}+y v_{i-2} \quad(2 \leqq i \leqq n) .
\end{gathered}
$$

Notice $v_{r}$ has term of highest degree $x^{\prime}$. We need also the following result:

Lemma 3.5. Let $z=x^{2}+2 y$ and $r=2 p$ or $2 p+1$, then

$$
z^{r} y^{n-2-r}=\left(\begin{array}{l}
r \\
p
\end{array}\right) y^{n-2}, \quad(0 \leqq r \leqq n-2) .
$$

Proof. First we claim that $z^{r} y^{n-2-r}$ is identically equal to

$$
\begin{aligned}
v_{2 r} y^{n-2-r}+v_{2 r-2} y^{n-r-1}+r\left(v_{2 r-4} y^{n-r}\right. & \left.+v_{2 r-6} y^{n-r+1}\right) \\
& +\left(\begin{array}{l}
r \\
2
\end{array}\right)\left(v_{2 r-8} y^{n-r+2}+v_{2 r-10} y^{n-r+3}\right)+\ldots
\end{aligned}
$$

the sum finishing with

$$
\ldots+\left(\begin{array}{c}
r \\
p-1
\end{array}\right)\left(v_{4} y^{n-4}+v_{2} y^{n-3}\right)+\left(\begin{array}{l}
r \\
p
\end{array}\right) y^{n-2}
$$


if $r=2 p$, and

For $r=1$, we have

$$
\ldots+\left(\begin{array}{l}
r \\
p
\end{array}\right)\left(v_{2} y^{n-3}+y^{n-2}\right) \quad \text { if } \quad r=2 p+1 .
$$

$$
z y^{n-3}=\left(x^{2}+2 y\right) y^{n-3}=\left(v_{2}+y\right) y^{n-3}=v_{2} y^{n-3}+y^{n-2} .
$$

Now we proceed by induction, showing that the result for case $r=2 p-1$, $p \geqq 1$, implies case $r=2 p$, which in turn implies case $r=2 p+1$. This all follows from (3.4). To complete the proof of the Lemma, apply relations (3.2).

\section{Chern classes and the normal bundle}

Our object in this section is to express $C(c(v))$ in terms of the generators of the cohomology of $G_{n}$, namely the Chern classes of the canonical bundle $\gamma(3.1)$.

It is not hard to show that the tangent bundle of $G_{n}$ as a complex manifold is (isomorphic to) Hom $(\gamma, \lambda)$, the bundle whose fibre over a given point in $G_{n}$ is the space of homomorphisms of the fibre of $\gamma$ into that of $\lambda$. A similar result holds for Grassmannians in general (see (6), p. 411). Now since $\gamma \oplus \lambda=n$ (see §2) and $\operatorname{Hom}(\gamma, \lambda)=\gamma^{*} \otimes \lambda(3$, p. 47), we have

$$
\operatorname{Hom}(\gamma, \lambda) \oplus\left(\gamma^{*} \otimes \gamma\right)=\gamma^{*} \otimes n=n \gamma^{*}
$$

The tangent bundle $\tau$ itself is the decomplexification of $\operatorname{Hom}(\gamma, \lambda)(3, \mathrm{p}, 67)$. Thus an elementary calculation, using " $c r=1+*$ " and other basic relations between bundles (3, p. 47 and 5 , p. 242), shows

$$
c(\tau) \oplus 2\left(\gamma^{*} \otimes \gamma\right)=n\left(\gamma^{*} \oplus \gamma\right) .
$$

The triviality of $\tau \oplus v$ implies that of $c(\tau) \oplus c(v)$, and we apply (2.1) to this and the formula above to obtain

$$
C(c(v))=\left(C\left(\gamma^{*} \otimes \gamma\right)\right)^{2} \cdot\left(C\left(\gamma^{*} \oplus \gamma\right)\right)^{-n} .
$$

Next, using the method of (3), p. 64, we write formally

$$
\sum_{i=0}^{2} c_{i}(\gamma) t^{i}=(1+a t)(1+b t)
$$

with $t$ as indeterminate. Then

$$
\sum_{i=0}^{2} c_{i}\left(\gamma^{*}\right) t^{i}=(1-a t)(1-b t)
$$

and with $x=-(a+b), y=-a b(\S 3)$, we have

$$
\begin{aligned}
& \sum_{i=0}^{4} c_{i}\left(\gamma^{*} \oplus \gamma\right) t^{i}=\left(1-a^{2} t^{2}\right)\left(1-b^{2} t^{2}\right)=1-\left(x^{2}+2 y\right) t^{2}+y^{2} t^{4}, \\
& \sum_{i=0}^{4} c_{i}\left(\gamma^{*} \otimes \gamma\right) t^{i}=1-(a-b)^{2} t^{2}=1-\left(x^{2}+4 y\right) t^{2} .
\end{aligned}
$$


Observe that no odd power of $t$ appears in the two formulae above. In fact, substituting these in (4.1), replacing $t^{2}$ by the indeterminate $T$, and setting $z=x^{2}+2 y$, we have finally

Lemma 4.2. For the complexified normal bundle of $G_{n}$, the odd Chern classes $c_{2 i+1}$ are zero. The class $p_{i}(v)$ is the coefficient of $T^{i}$ in

$$
(1+(z+2 y) T)^{2}\left(1+z T+y^{2} T^{2}\right)^{-n} \text {. }
$$

Remark. $c_{2 i+1}=0$ is also implied by the fact that in general the odd Chern classes of a complexified real bundle have order $2(5$, p. 243), whereas $H^{*}\left(G_{n}\right)$ is torsion-free (3.1). It means nothing is lost by the exclusion of these classes in Proposition 2.2.

\section{Proof of the Main Theorem}

According to Proposition 2.2, the Main Theorem 1.1 will follow if we can show the coefficient of $T^{n-2}$ in Lemma 4.2 is non-zero. Define the weight of the monomial $x^{r} y^{s} \in H^{2 r+4 s}\left(G_{n}\right)$ to be $r+2 s$, then all monomials of weight greater than $2 n-4$ vanish, since $H^{i}\left(G_{n}\right)=0$ for $i>4 n-8$ (the dimension of $G_{n}$ ). Clearly $T^{r}$ appears with coefficient of weight $2 r$. Now the only generator of weight $2 n-4$ is $y^{n-2}$, so that $T^{n-2}$ has coefficient $D \cdot y^{n-2}$ for some integer $D$. Therefore we must prove $D$ is non-zero. Since higher powers of $T$ vanish, Theorem 1.1 is the sharpest result obtainable by using Proposition 2.2.

Let $\left(1+z T+y^{2} T^{2}\right)^{-n}=\Sigma d_{i} T^{i}(0 \leqq i \leqq n-2)$, then, from Lemma 4.2,

$$
D y^{n-2}=d_{n-2}+2(z+2 y) d_{n-3}+(z+2 y)^{2} d_{n-4} \text {. }
$$

The binomial expansions of the various powers of $z T+y^{2} T^{2}$ yield the $d_{i}$ 's required.

We evaluate $D$ with the aid of Lemma 3.5. Set

$$
S(L, m, s)=\sum_{k=0}^{t-L}(-1)^{k}\left(\begin{array}{c}
4 t-k-m-1 \\
2 t-k-m
\end{array}\right)\left(\begin{array}{c}
2 t-k-m \\
2 t-2 k-m
\end{array}\right)\left(\begin{array}{c}
2 t-2 k-s \\
t-k-\left[\frac{1}{2} s\right]
\end{array}\right),
$$

where $\left[\frac{1}{2} s\right]$ means the integral part. Let $S^{\prime}$ be obtained by replacing $4 t-k-m-1$ by $4 t-k-m$. Then for $n=2 t$, we obtain

$$
D=S(1,2,2)-2 S(2,3,2)-4 S(2,3,3)+S(2,4,2)+4 S(2,4,3)+4 S(2,4,4) \text {. }
$$

For $n=2 t+1$,

$$
-D=S^{\prime}(1,1,1)-2 S^{\prime}(1,2,1)-4 S^{\prime}(1,2,2)+S^{\prime}(2,3,1)
$$

After some calculation, we obtain

$$
+4 S^{\prime}(2,3,2)+4 S^{\prime}(2,3,3) \text {. }
$$

$$
\begin{aligned}
& D=\left(\begin{array}{c}
3 t-3 \\
t-2
\end{array}\right)^{2} \cdot \frac{t^{2}-8 t+6}{(t-1)^{2}} \quad(n=2 t) \\
& D=-3\left(\begin{array}{c}
3 t-1 \\
t-1
\end{array}\right)\left(\begin{array}{c}
3 t-2 \\
t-2
\end{array}\right) \frac{t^{2}-9 t+6}{(t-1)(3 t-2)} \quad(n=2 t+1) .
\end{aligned}
$$


Since the equations $t^{2}-8 t+6=0$ and $t^{2}-9 t+6=0$ have no integer solutions, $D$ is non-zero for $t \geqq 2$, and the proof of the Main Theorem is complete.

In conclusion, I would like to thank the referee for his helpful comments.

\section{REFERENCES}

(1) M. F. AtrYaH, Immersions and embeddings of manifolds, Topology 1 (1962), 125-132.

(2) A. Borel, Sur la cohomologie des espaces fibrés principaux et des espaces homogènes de groupes de Lie compacts, Ann. of Math. (2) 57 (1953), 115-207.

(3) F. HIRZEBRUCH, Topological methods in algebraic geometry (translated by R. L. E. Schwarzenberger; Springer-Verlag, 1966).

(4) S. G. Hoggar, On KO-theory of Grassmannians, Quart. J. Math. Oxford Ser. (2) 20 (1969), 447-463.

(5) D. Husemoller, Fibre bundles (McGraw-Hill, 1966).

(6) I. R. Porteous, Topological geometry (Van Nostrand, 1969).

(7) B. J. Sanderson, Immersions and embedding of projective spaces, Proc. London Math. Soc. (3) 14 (1964), 137-153.

(8) H. WhITNEY, The self intersections of a smooth manifold in $2 n$-space, Ann. of Math. (2) 45 (1944), 220-246.

(9) H. WhITnEy, The singularities of a smooth $n$-manifold in $(2 n-1)$-space, Ann. of Math. (2) 45 (1944), 248-293.

UNIVERSITY OF WARWICK

AND

UNIVERSITY OF GLASGOW 